\title{
Study of electrical discharge machining technology for slicing silicon ingots
}

\author{
W.Y. Peng, Y.S. Liao* \\ Department of Mechanical Engineering, National Taiwan University, Taipei, Taiwan, ROC
}

\begin{abstract}
Silicon slicing technology is an undergoing process and its performance improvements meet the ever-challenging and versatile demands. A new attempt to apply the WEDM strategy to slice the semiconductor materials is studied. The barriers from unusual material characteristics are to be conquered to make this idea realizable. The existing WEDM technology is utilized to slice the heavy-doped silicon ingot and its feasibility is examined. The machining rate and surface roughness are measured under various current on times and servo voltages in both the water immersed and water flushing WEDM machines. If small current on time is collocated with proper off time and lower gap voltage sensitivity under automatic feed mode, the stable area machining rate of around $76 \mathrm{~mm}^{2} / \mathrm{min}$ can be attained, and the $R_{\mathrm{a}}$ value is $3.6 \mu \mathrm{m}$ or so which is acceptable if the following polishing procedure is considered. The thickness of defects to be polished can be predicted from the SEM photographs of the cross-sections of the sliced wafers. If the wire diameter is $0.25 \mathrm{~mm}$ and the wafer thickness is $1 \mathrm{~mm}$, the portion of material loss including the kerf and the amount to be polished is under $26 \%$.
\end{abstract}

(C) 2003 Elsevier B.V. All rights reserved.

Keywords: WEDM; Silicon ingot; Servo voltage; Surface roughness; Current on time; Current off time

\section{Introduction}

Monocrystalline silicon is now the most commonly used substrate material for the production of microelectronic components worldwide. Conventional inner diameter (ID) saws and wire saws are two main equipments used for slicing silicon ingots. These equipments adopt contact force to slice the ingots that easily results in harmful surface micro-cracks or other damages to the wafer due to mechanical abrasive nature. Chonan et al. [1] analyzed the stress of silicon-wafer slicer. The stress comes from the tension of wire, spinning, and loading from the workpiece. The vibration and deflection of a silicon-wafer slicer [2] is further studied and simulated but the improvement in performance is limited. Additionally, ID saw deteriorates throughput when it is used for slicing large silicon ingots.

Wire saw technology is now replacing ID saw slicing technology. Li et al. [3] investigated the modeling of contact stress. This equipment, especially the multi-wire saw is widely adopted today, but the process has problems relating to dirty work environment, low efficiency and instability from the wire system [4]. Besides, smaller wafer thickness is

\footnotetext{
* Corresponding author. Fax: +886-2-23660443.

E-mail address: liaoys@ntu.edu.tw (Y.S. Liao).
}

profitable because of the high material cost. The wafer size becomes larger to accommodate more chips. This also makes the slicing process an important topic to be re-examined and re-investigated.

Meanwhile, methodically speaking, the improvement can be achieved from these existing methods or a new procedure of brand new ideas or modifications of other existing technology. The wire electrical discharge machining (WEDM) technology is adopted as a new candidate for silicon slicing to be compared with the existing procedures and testified if it can inevitably substitute for the ID saw or even compete with wire saws in some manufacturing orders.

It was implemented by Luo et al. [5] in 1995 to use WEDM of rapid-wire type to slice heavy-doped silicon ingots. Thin and little geometrical error wafers are produced with this process. High contact resistance on the ingot surface is solved by nickel-plating. The surface roughness, cutting efficiency and micro-structures under different energy intensities are observed. But the effects of this procedure are not overall well demonstrated.

WEDM is a kind of well-developed technology nowadays. No matter the surface slicing rate, surface roughness or stability problem are almost well solved. In which, water immersed and water flushing types are two main machine categories used worldwide, and they have been proved to have better manufacturing efficiency and quality than those 
of the rapid-wire type. If the WEDM technology may be utilized in silicon slicing process, both these kinds of machines should be selected to conduct the related experiments. The former machines use de-ionized water as the dielectric fluid and their wires are not used repeatedly, but the rapid-wire type uses kerosene as the dielectric fluid and the wire is used repeatedly. Since they have quite much difference on power source characteristics and configurations of hardware, different problems and phenomena may be encountered and surely the procedures adopted for manufacturing processes should be reconsidered. The results of the sliced wafers are examined under different operation conditions, such as current on time, current off time, water pressure and wire tension. It is overall compared with the wire saw and ID saw on the kerf ratio, average cutting speed and surface roughness.

\section{Methodology and procedure}

\subsection{Material characteristics and preview discussion}

For monocrystalline silicon, its melting point is about $1400{ }^{\circ} \mathrm{C}$ which is close to that of normal carbon steels and its thermal conductivity is about $150 \mathrm{~W} / \mathrm{m}^{\circ} \mathrm{C}$ which is similar to borosilicate glass. Besides, its specific heat is $0.7 \mathrm{~J} / \mathrm{g}{ }^{\circ} \mathrm{C}$. However, the current conduction phenomena in the semiconductor material under a temperature field especially in WEDM machining mechanism, is quite complex. It is hard to predict how high the local temperature is and observe how the p-type ingot melts near the discharge wire. Anyhow, the temperature gradient can be quite steep at the local sparking area and there would be moderate temperature rise of the whole ingot out of the inner resistance which generates heat after current passes through.

In the experiment, the $3 \mathrm{in.} C Z\langle 111\rangle$ oriented silicon ingot is p-type with $30 \Omega \mathrm{cm}$ resistivity. Because it is a new attempt in this experiment to cut the semiconductor material with the WEDM machine, problems out of the different characteristics of medium or heavy-doped silicon ingots should be checked or tested under the machine first. It is found that the temperature rise after a period of the discharge reduces the resistivity and hence abnormal discharges. This comes from the conductivity elevation effects of semiconductors under the higher temperature. In order to keep the wafer of correct orientation, a fixture is designed to enable easily fix the ingot as shown in Fig. 1. It is also observed that breakage at the wafer rim often occurs out of the flushing water and the explosive tension forces of intensive discharge. A protective and supporting end cap is integrated into the fixture to avoid destroying the newly produced wafer. The local sketch of the ingot with the fixture on both the machine types of water flushing type and water immersed type are, respectively, shown in Figs. 2 and 3.

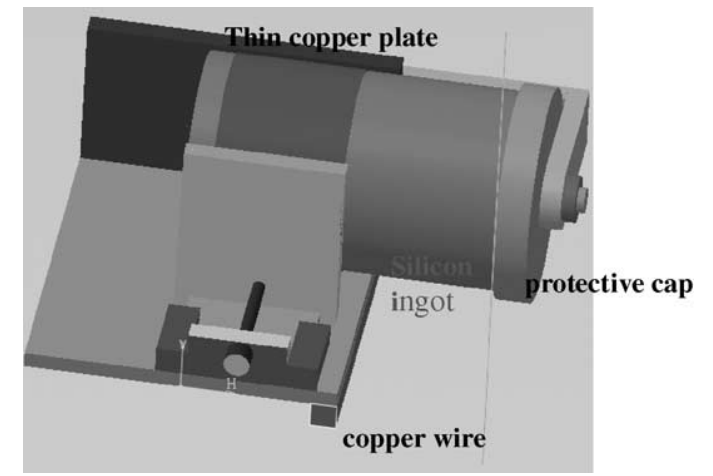

Fig. 1. Fixture to orient the ingot and protect the thin wafer.

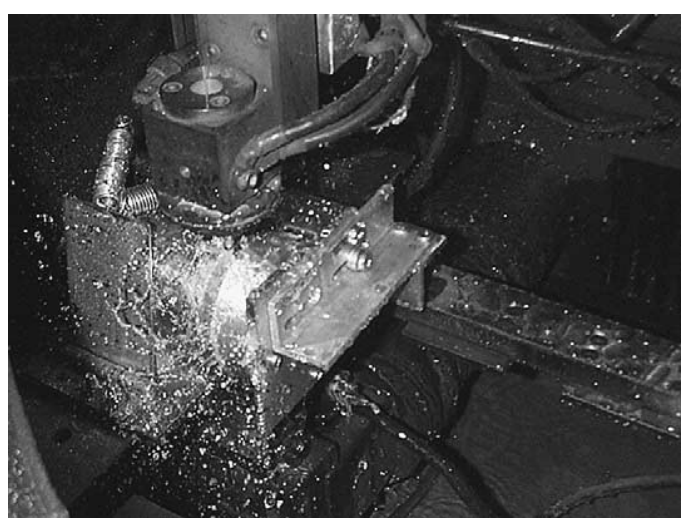

Fig. 2. WEDM machine of water flushing type to slice silicon ingot with fixture used.

\subsection{Operation requirements and implementation policy}

Since continuous discharge is possible, the cutting efficiency and quality of the wafer are further concerned. There are two modes of feed, automatic mode and manual mode, in the WEDM machine. The thickness of workpiece varies when cutting process proceeds especially at the beginning stage of slicing since the cross-section is circular. Being able to slice one piece of wafer smoothly without wire breakages, the mode of manual feed rate is adopted first and the servo

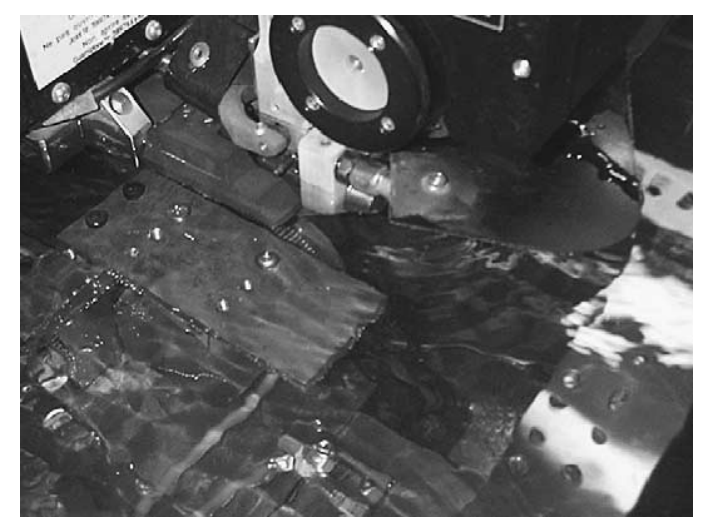

Fig. 3. WEDM machine of water immersed type. 
feed mode follows in the NC program. This operation can be simplified if the controller is intelligent enough, like the AGIE 500 or further series, it can adjust its servo parameters after the occurrence of some wire breakages. The water flushing and water immersed types of WEDM machines are taken to conduct the experiments under different operation conditions and their whole performances are compared with each other.

Under cutting process, current on time is most usually adjusted together with the current off time. These adjustments influence the energy intensities and the effects can be easily observed on surface roughness and cutting speed. What energy intensity order in WEDM suits the semiconductor material of certain doping rate is mainly concerned. There can be some optimum or acceptable combinations of parameters after some experiments are conducted. The influence of other parameters, like wire tension and its speed, which are not so primary to the cutting efficiency and quality in general WEDM manufacturing would also be observed.

The kerf is measured with gages under different current on time. The micro-structure of the wafer is observed with SEM. These photographs help to evaluate the thickness of surface layer to be polished and check if there is any harmful micro-crack on the wafer.

\section{Results and discussions}

\subsection{Slicing efficiency and stability}

The total efficiency related to the stability of feed rate and average cutting speed. No matter under automatic servo mode or manual mode, certain gap voltage should be maintained to keep effective discharges. The average gap voltage is a gross method but a useful control strategy in most traditional WEDM machines. Due to different material characteristics of silicon ingots comparing to steels of good conductance, the gap voltage should be set at higher value to avoid accidental touch of the wire with the ingot that results in wire breakage or abrasive damage to the wafer surface as shown in Fig. 4. In WEDM machining, efficiency is often evaluated by surface slicing rate which is the product of the feed rate and the workpiece thickness. The data of feed rate and the cut length of each moment is recorded to derive the variation of surface slicing rate during the slicing process. The time taken to slice a single wafer is referenced to compute the average cutting speed.

The energy level is related to current on time since the peak current is the product of the on time and the current rising slope. Taking the water flushing type machine in this experiment for example, if the on time is set to $0.8 \mu \mathrm{s}$, and the slope is about $450 \mathrm{~A} / \mu \mathrm{s}$, then the peak current would be 360 A. From the current signal graphs on the oscilloscope, higher ratio of abnormal discharge than usual situations oc-

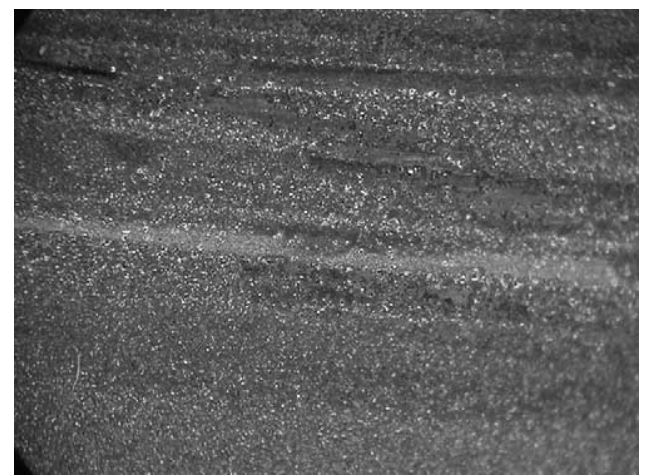

Fig. 4. Surface damage due to wire breakage or abrasive effect from wire touching.

Table 1

Instantaneous surface slicing rate under fixed current off time $=2.25 \mu \mathrm{s}$

\begin{tabular}{llllll}
\hline \multicolumn{7}{c}{ On time $(\mu \mathrm{s})$} \\
\cline { 2 - 6 } & 0.2 & 0.3 & 0.4 & 0.5 & 0.6 \\
\hline $\begin{array}{c}\text { Instantaneous surface } \\
\text { slicing rate }\left(\mathrm{mm}^{2} / \mathrm{min}\right)\end{array}$ & 27.2 & 39.2 & 43.9 & 54.6 & 90.8 \\
\hline
\end{tabular}

Table 2

Instantaneous surface slicing rate under fixed current off time $=1.8 \mathrm{~s}$

\begin{tabular}{clllll}
\hline \multicolumn{7}{c}{ On time $(\mu \mathrm{s})$} \\
\cline { 2 - 6 } & 0.2 & 0.3 & 0.4 & 0.5 & 0.6 \\
\hline $\begin{array}{c}\text { Instantaneous surface } \\
\text { slicing rate }\left(\mathrm{mm}^{2} / \mathrm{min}\right)\end{array}$ & 33.6 & 40.4 & 54.6 & 79.5 & 100.4 \\
\hline
\end{tabular}

curs. This proves that the feed rate cannot be as high as cutting general steel materials.

The current on time, off time and sensitivity of gap voltage under automatic servo mode should be adjusted to proper values to minimize the proportion of abnormal discharges. The cutting speed symbolizes the material removal rate and obviously is related to the energy intensity. The current on time and off time together mainly decide the energy release intensity. Tables 1 and 2 and Fig. 5 show the instantaneous surface slicing rate under different current on time and off time of the water flushing type of WEDM machine. If the

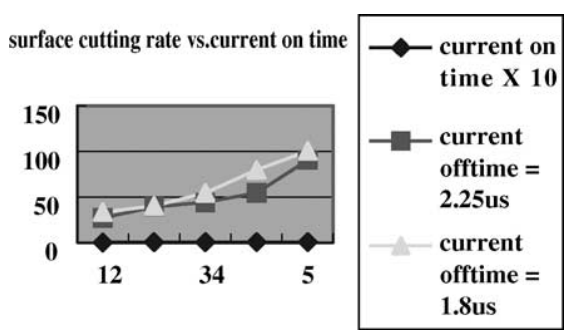

Fig. 5. Instantaneous surface slicing rate under different discharge intensities. 


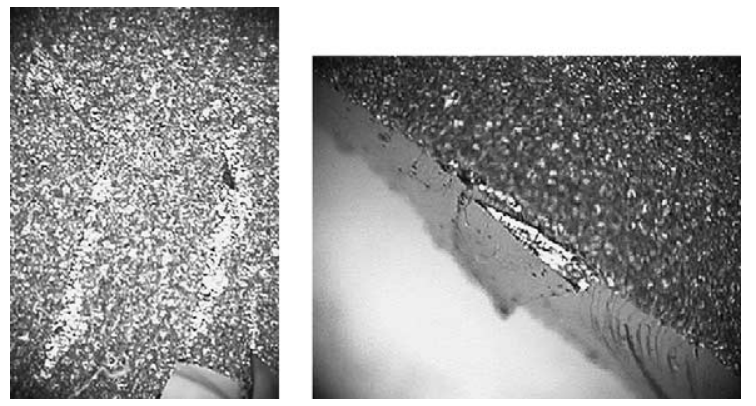

Fig. 6. Rim breakage of wafer.

current on time is too large or the off time is too short, wire breakage due to too much heat release or explosive power may easily occur. A current on time about $0.5 \mu \mathrm{s}$ with a current off time of $1.8 \mu$ s or so but not less than $1.1 \mu$ s attains stable and efficient performance of the slicing task.

Besides, the upper wire guide of the WEDM machine is kept at fixed height during slicing operation since the cross-section is circular and the upper nozzle cannot bump the workpiece, so flushing effects are not well at most of the time. But when the wire approaches the highest point of the ingot, the flushing effect becomes better and surface slicing rate evidently improves during that short period of time. This problem almost disappears in water immersed type of WEDM machine and therefore the overall slicing efficiency improves about 28-33\%. The total time to slice one piece of wafer without further surface finishing by lower discharge power by water immersed type of WEDM machine is about 45-73 min. It depends on how quick and how rough of the surface are demanded.

\subsection{Surface roughness and geometric errors}

It has been mentioned previously that there may be breakage of the wafer rim which is shown in Fig. 6. The surface roughness after WEDM machining is good enough only when the discharge energy is low enough or repeated surface finishing operations are conducted. The surface roughness, $R_{\mathrm{a}}$, is at best $4.6 \mu \mathrm{m}$ or so for water flushing type after slicing without surface finishing operation. The surface roughness, $R_{\mathrm{a}}$, varies along the cutting direction since the water flushing effects and slicing rate are not the same. After one time of surface finishing operation, the $R_{\mathrm{a}}$ value is about $2 \mu \mathrm{m}$ or so. A surface texture of a sliced wafer by water immersed type WEDM machine, shown as Fig. 7, was evaluated over a length of $1 \mathrm{~mm}$. The surface experienced finishing operation one time. The maximum peak to valley height of the profile $R_{\mathrm{t}}$ was $8.5 \mu \mathrm{m}$, and the arithmetic mean of the roughness profile $R_{\mathrm{a}}$ was $1.34 \mu \mathrm{m}$.

Generally speaking the surface roughness mainly depends on the current on time. Tables 3 and 4 show the variation of surface roughness under various current on times. Figs. 8 and 9, the microscope and SEM photographs of the sliced

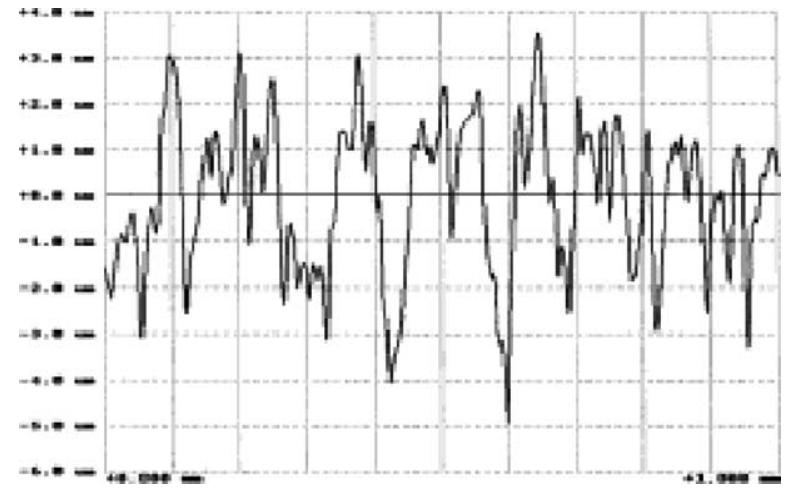

Fig. 7. Surface texture of the wafer sliced by the water immersed type of WEDM.

Table 3

Surface roughness $\left(R_{\mathrm{a}}\right)$ vs. on time (water flushing type of WEDM)

\begin{tabular}{llllll}
\hline & \multicolumn{5}{l}{ On time $(\mu \mathrm{s})$} \\
\cline { 2 - 6 } & 0.1 & 0.2 & 0.3 & 0.4 & 0.5 \\
\hline$R_{\mathrm{a}}(\mu \mathrm{m})$ & 4.6 & 5.8 & 7.2 & 9.0 & 10.6 \\
\hline
\end{tabular}

Table 4

Surface roughness $\left(R_{\mathrm{a}}\right)$ vs. on time (water immersed type of WEDM)

\begin{tabular}{llll}
\hline & \multicolumn{2}{l}{ On time $(\mu \mathrm{s})$} & \\
\cline { 2 - 4 } & 0.05 & 0.15 & 0.3 \\
\hline$R_{\mathrm{a}}(\mu \mathrm{m})$ & 3.8 & 4.4 & 5.6 \\
\hline
\end{tabular}

wafers, display the surface textures of different energy orders. The former has more vivid surface craters. The rough texture was produced by discharges of higher peak current. The craters are shallower when the peak current is smaller
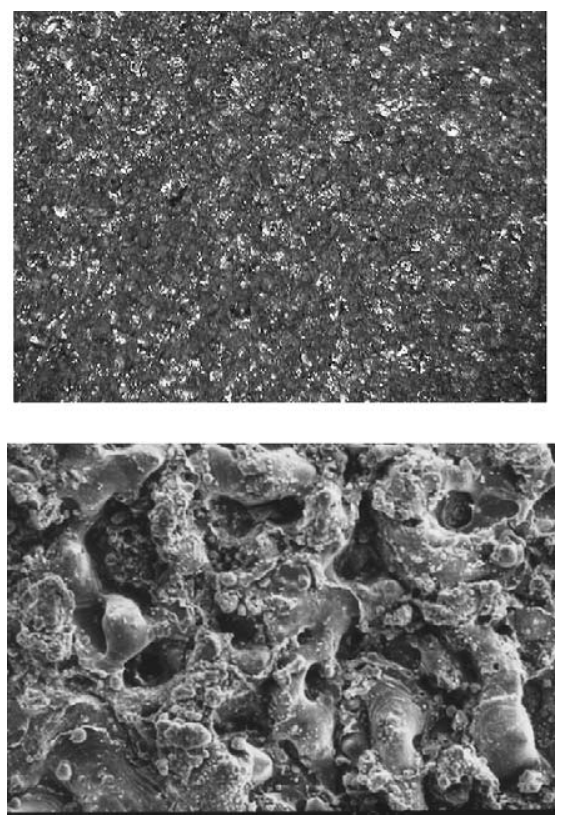

Fig. 8. Surface texture of the wafer with $R_{\mathrm{a}}=8.4 \mu \mathrm{m}$. 

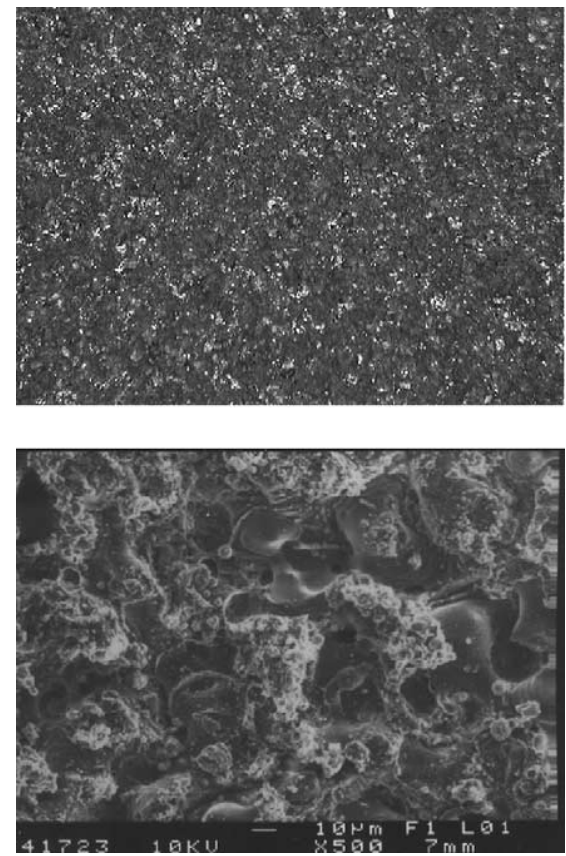

Fig. 9. Surface texture of the wafer with $R_{\mathrm{a}}=4.6 \mu \mathrm{m}$

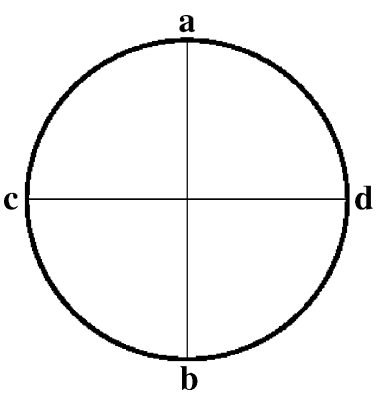

Fig. 10. Measurement paths of the surface profile ( $\mathrm{ab}$ and $\mathrm{cd}$ ).

which attains smaller $R_{\mathrm{a}}$ value. The total thickness variation (TTV) and profile bending rate, which is defined as warp, are also highly concerned in wafer slicing because it directly relates to the material waste rate and polishing time. Fig. 10 shows two measurement paths passing the center of the wafer surface. Line $a b$ is the vertical measurement

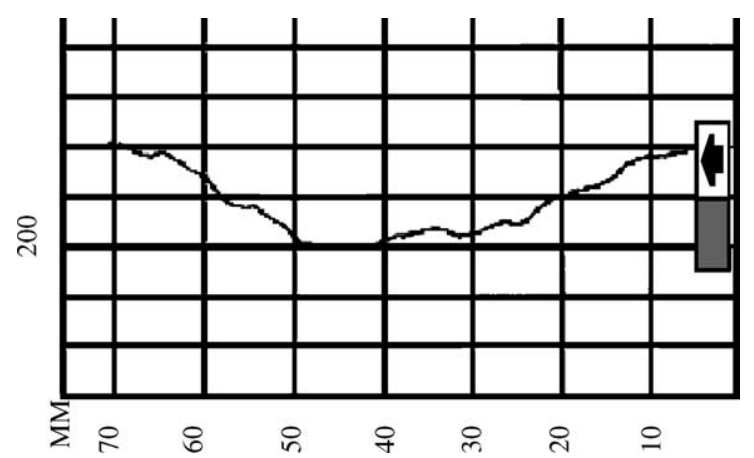

Fig. 11. Surface profile on the vertical path.

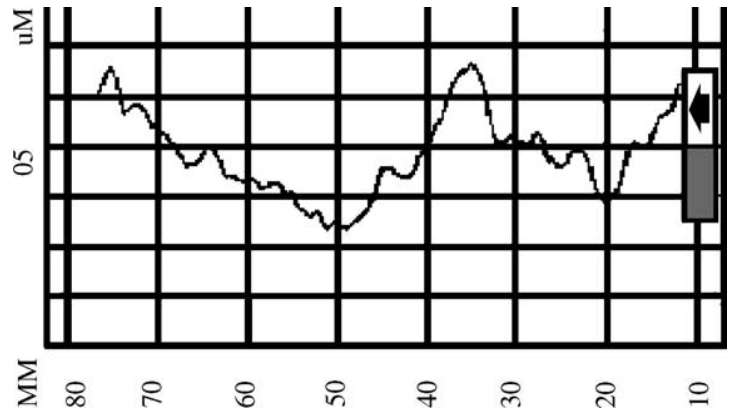

Fig. 12. Surface profile on the horizontal path.

path and line cd the horizontal one. The concave contour as shown in Fig. 11, the profile measurement along the vertical path, was manifest. The difference in height across the $65 \mathrm{~mm}$ traveling length was $4.3 \mu \mathrm{m}$, because the electrode wire vibrates wildly at the midpoint of the two diamond wire guides. The cutting wire, whose traveling speed was set at $90 \mathrm{~mm} / \mathrm{min}$, was maintained at a tension of approximately $12 \mathrm{~N}$.

The geometric error of drum shape may be a little reduced by enlarging the wire tension, but there still would exist certain extent of warp. The horizontal profile as shown in Fig. 12 appeared a $1.7 \mu \mathrm{m}$ error along the cd path of the sliced wafer. The TTV, which is within $5 \mu \mathrm{m}$ is quite small and it is far better than that by the wire saw production. According to the latest report of the wire saw technology, the TTV is still above $9.5 \mu \mathrm{m}$ or so. The warp extent in general is a little lower than that by the wire saw.

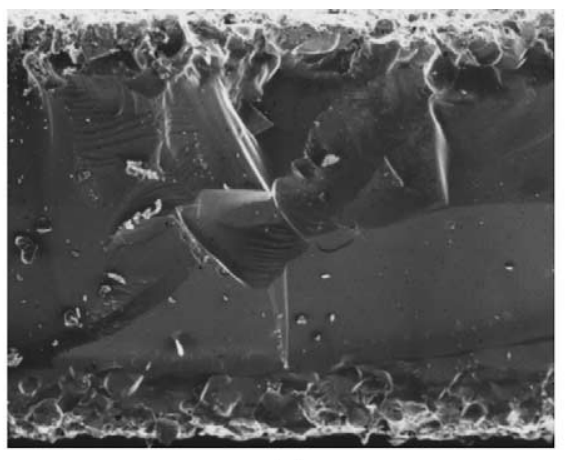

(a)

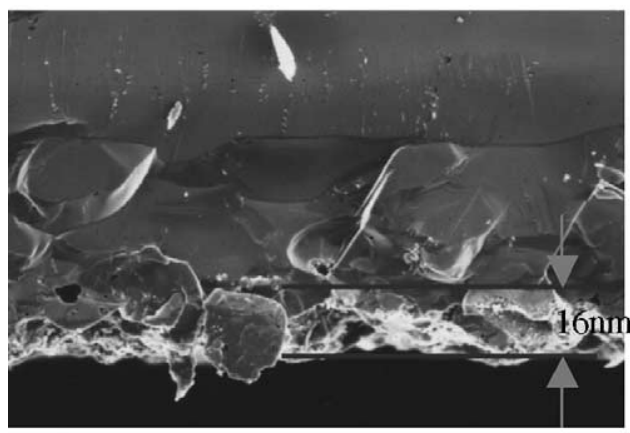

(b)

Fig. 13. The SEM photograph of the cross-section. 
Table 5

General comparisons of three slicing methods

\begin{tabular}{|c|c|c|c|}
\hline & Wire saw & ID saw & Wire EDM \\
\hline Slicing strategy & Abrasive machining of free particles & Adhesive diamond particles & Discharge energy \\
\hline Surface texture & Saw marks & Chipping and fracture & Discharge crater \\
\hline Defect thickness to be polished $(\mu \mathrm{m})$ & $25-35$ & $35-40$ & $15-26$ \\
\hline Area production rate $\left(\mathrm{cm}^{2} / \mathrm{h}\right)$ & $110-220$ & $20-40$ & $45-65$ \\
\hline Minimum wafer thickness $(\mu \mathrm{m})$ & 250 & 350 & 250 \\
\hline Accommodative ingot diameter (mm) & 300 & 200 & 200 \\
\hline Warp & Slightly & Severe & Slightly \\
\hline Time to slice 3 in. ingot (h) & $4.5-6.5$ & $2.5-4$ & $1.2-2$ \\
\hline Surface roughness, $R_{\mathrm{a}}(\mu \mathrm{m})$ & $2-4$ & $3-5$ & $3-8$ \\
\hline $\operatorname{Kerf}(\mu \mathrm{m})$ & $150-210$ & $300-500$ & 280-290 (0.25 mm wire) \\
\hline Average material waste $(0.7 \mathrm{~mm}$ thick wafer $)(\%)$ & 26 & 42 & 38 \\
\hline
\end{tabular}

\subsection{Kerf and total material waste}

The thickness of defects which needs to be polished can be predicted from the SEM photograph of the cross-section of the sliced wafer. In Fig. 13, the predicted value is about $16 \mu \mathrm{m}$, which is quite acceptable. The kerf is related to the wire diameter and the discharge energy level. The kerf is $0.289 \mathrm{~mm}$ or so if a $0.25 \mathrm{~mm}$ copper wire is used with large current on time. It can be $0.22 \mathrm{~mm}$ or so if a $0.2 \mathrm{~mm}$ molybdenum wire is used. Considering the wafer thickness, kerf and the predicted thickness of surface defect, the total material waste rate can be estimated. In average, it is within $28 \%$. Table 5 is the general comparison of the three strategies for slicing ingots. Due to some fundamental differences of these three machining methods, not all the conditions are assumed to be even. At least, the non-contact slicing scheme does reduce the geometric errors of the wafer. If the total batch production rate should compete with that of a wire saw system, the WEDM may somehow need a multi-wire system, three or five wires parallel, with separate discharge power control.

\section{Conclusions}

It is verified that the properly doped ingot can be sliced by WEDM method. As long as the discharge parameters match the material characteristics, the wire breakage can be avoided and the slicing efficiency is acceptable if the demand of wafer amount is small. The surface roughness is reasonable and the geometric errors are quite satisfactory. Generally speaking, this method can be a substitutive choice for current wire saw or ID saw machines in certain situations. However, this strategy does not suit for the highly pure silicon ingot so far.

\section{References}

[1] S. Chonan, M.Y. Jiang, L. Yukiy, Stress analysis of a silicon-wafer slicer cutting the crystal ingot, J. Mech. Des. 115 (1993) 517-711.

[2] S. Chonan, M.Y. Jiang, L. Yukiy, Vibration and defection of a silicon-wafer slicer cutting the crystal ingot, J. Vibr. Acoust. 115 (1993) 529-534.

[3] J. Li, I. Kao, V. Prasad, Modeling stresses of contacts in wire saw slicing of polycrystalline and crystalline ingots: application to silicon wafer production, J. Electron. Packaging 120 (1998) 123-128.

[4] Y. Shimazaki, Y. Tani, M. Suzuki, Y. Kanda, Development of a resinoid diamond wire containing metal powder for slicing a slicing ingot, Ann. CIRP 48 (1) (1999) 273-276.

[5] Y.F. Luo, C.G. Chen, Z.F. Tong, Investigation of silicon wafering by wire EDM, J. Mater. Sci. 27 (1992) 5805-5810. 